University of Nebraska - Lincoln

DigitalCommons@University of Nebraska - Lincoln

Faculty Publications from Nebraska Center for Materials and Nanoscience, Nebraska Center Materials and Nanoscience

$5-15-2002$

\title{
Structure of macrodomain walls in polytwinned magnets
}

Kirill D. Belashchenko

University of Nebraska-Lincoln, belashchenko@unl.edu

Vladimir P. Antropov

Ames Laboratory, US Department of Energy, antropov@ameslab.gov

Follow this and additional works at: https://digitalcommons.unl.edu/cmrafacpub

Part of the Nanoscience and Nanotechnology Commons

Belashchenko, Kirill D. and Antropov, Vladimir P., "Structure of macrodomain walls in polytwinned magnets" (2002). Faculty Publications from Nebraska Center for Materials and Nanoscience. 23. https://digitalcommons.unl.edu/cmrafacpub/23

This Article is brought to you for free and open access by the Materials and Nanoscience, Nebraska Center for (NCMN) at DigitalCommons@University of Nebraska - Lincoln. It has been accepted for inclusion in Faculty Publications from Nebraska Center for Materials and Nanoscience by an authorized administrator of DigitalCommons@University of Nebraska - Lincoln. 


\title{
Structure of macrodomain walls in polytwinned magnets
}

\author{
K. D. Belashchenko a) and V. P. Antropov \\ Ames Laboratory, Ames, Iowa 50011
}

We propose a microscopic approach to the studies of magnetic configurations in hard magnets which may be conveniently used for nanoscale systems; the microstructure of the magnet is easily and naturally included in the calculations. This approach is applied to find the structure of macrodomain walls in polytwinned magnets of the CoPt family. Magnetostatic fields are small compared to the anisotropy field in these magnets; direct simulation shows that in this case the macrodomain wall is not continuous, but rather comprised of segments held together by relatively small magnetostatic forces. This segmentation is expected to have a strong effect on magnetization processes. (C) 2002 American Institute of Physics. [DOI: 10.1063/1.1453323]

It is known that the hysteretic properties of a magnet are sensitive to microstructure, because the domain wall (DW) motion and domain nucleation are strongly affected by lattice imperfections. The majority of hard magnets are based on rare earth-transition metal alloys $(\mathrm{Nd}-\mathrm{Fe}-\mathrm{B}$ type and $\mathrm{Sm}-\mathrm{Co}$ type), ${ }^{1,2}$ where microstructure is dominated by grain boundaries, sometimes with segregated intergranular phases. The role of these defects was studied in a number of papers using the finite-element version of the micromagnetic method. ${ }^{3,4}$

There is another group of intermetallic permanent magnets with high uniaxial anisotropy in the tetragonal $\mathrm{L}_{0}$ phase including $\mathrm{CoPt}$, FePt, and FePd. Unlike other permanent magnets, their microstructure is dominated not by grain boundaries, but by twin boundaries and antiphase boundaries. Theoretical understanding of the hysteretic properties of these magnets is rather poor.

In this article we study the structure of domain walls in CoPt type magnets. Microstructures of these magnets typically exhibit polytwinning, i.e., the formation of regular arrays (stacks) of ordered bands ( $c$ domains) separated by twin boundaries. ${ }^{5-9}$ The tetragonal axes $c$ in these domains (pointing along one of the three cubic axes of the parent fcc phase) alternate regularly making $90^{\circ}$ angles between the adjacent domains. The kinetics of polytwinning was studied using both the phenomenological ${ }^{8}$ and microscopical ${ }^{9}$ approaches, and the main features of the experimentally observed microstructures are well reproduced in these theoretical studies.

The magnetic structure of polytwinned magnets was studied for several decades using analytical micromagnetic methods. ${ }^{5}$ Due to high magnetic anisotropy the DW width $\delta$ is quite small $\left(50-100 \AA\right.$ ), while the anisotropy field $H_{a}$ $=2 K / M(K$ is the magnetocrystalline anisotropy constant, and $M$ is the saturation magnetization) significantly exceeds the magnetostatic fields $H_{m} \sim 4 \pi M$ (the magnetostatic parameter $\eta=2 \pi M^{2} / K$ is close to 0.1 in CoPt and FePt and 0.38 in $\left.\mathrm{FePd}^{5}\right)$. Therefore, at $d \gg \delta$ ( $d$ being the thickness of the $c$ domain, usually at least $20 \mathrm{~nm}$ ) each ordered twin may be regarded as an individual magnetic domain (with intrinsic $90^{\circ} \mathrm{DWs}$ at the twin boundaries). It is assumed that magnetization processes are associated with macrodomain walls (MDW) crossing a large number of twins. Such MDWs were

\footnotetext{
${ }^{a)}$ Electronic mail: kdbel@ameslab.gov
}

observed experimentally, ${ }^{5,7}$ but their internal structure is unknown. ${ }^{5}$ This structure is crucial for hysteretic properties, and we will explore it using a microscopical approach which is convenient at the relevant length scale of up to $100 \mathrm{~nm}$, and which is especially useful when realistic microstructures must be incorporated in magnetic simulations.

We consider a magnetic alloy with the following classical Hamiltonian:

$$
\begin{aligned}
H= & H_{\mathrm{conf}}+\sum_{i<j} n_{i} n_{j}\left[-J_{i j} \boldsymbol{\mu}_{i} \boldsymbol{\mu}_{j}+\boldsymbol{\mu}_{i} \hat{D}_{i j} \boldsymbol{\mu}_{j}\right] \\
& +\sum_{i} n_{i}\left[E_{a}\left(\boldsymbol{\mu}_{i}\right)-\mathbf{H}_{0} \boldsymbol{\mu}_{i}\right],
\end{aligned}
$$

where $H_{\text {conf }}$ is the configurational part of the Hamiltonian which is independent on the magnetic state; $i$ and $j$ run over the lattice sites (and include the component index if there are several magnetic components); $n_{i}=1$ if site $i$ is occupied by a magnetic atom and $n_{i}=0$ otherwise; $\boldsymbol{\mu}_{i}$ is the (classical) magnetic moment of the atom at site $i ; J_{i j}$, the Heisenberg exchange parameter; $\mathbf{H}_{0}$, the external magnetic field; $E_{a}\left(\boldsymbol{\mu}_{i}\right)$, the magnetocrystalline anisotropy (MCA) energy equal to $-b_{i}\left(\boldsymbol{\mu}_{i} \mathbf{e}_{i}\right)^{2}$ in the case of an easy-axis anisotropy along $\mathbf{e}_{i}$; and $\hat{D}_{i j}$, the dipole-dipole interaction tensor. We assume that the magnetic moments are rigid $\left(\left|\boldsymbol{\mu}_{i}\right|=\mu\right)$, and the dynamics of the system is associated with their rotation.

The inhomogeneous and nonequilibrium states of the system may be described by the free energy

$$
F=\langle H+T \ln P\rangle,
$$

where $T$ is temperature, and $P$ is the distribution function. The full canonic statistical ensemble may not be used to describe the states with broken symmetry. For example, if $\mathbf{H}_{0}$ is zero, $H$ is invariant in respect to inversion of all spins, $\boldsymbol{\mu}_{i} \rightarrow-\boldsymbol{\mu}_{i}$, and all averages $\operatorname{Tr} \boldsymbol{\mu}_{i} P_{0}$ over the distribution function $P_{0}=\exp [\beta(F-H)]$ are zero. For inhomogeneous magnetic states with a fixed atomic configuration, let us introduce the "generalized Gibbs distribution" $P=\exp [\beta(\widetilde{F}$ $\left.\left.-H_{\text {eff }}\right)\right]$ as it was done ${ }^{10}$ for $H_{\text {conf }}$. In the theory of secondorder phase transitions the effective Hamiltonian $H_{\text {eff }}$ is a functional of the macroscopic order parameter field; ${ }^{11}$ in the micromagnetic approach ${ }^{12}$ this is the field of magnetization 
$\mathbf{M}(\mathbf{r})$. In our microscopic case $H_{\text {eff }}$ contains some effective parameters depending on the environment, i.e., on the average values of $\boldsymbol{\mu}_{i}$ and, if necessary, of their powers and correlators. In the simplest mean-field approximation (MFA) which will be used in this article, in equilibrium these parameters are the mean fields $\mathbf{H}_{i}=\mathbf{H}_{0}+\Sigma_{j}\left(J_{i j}-\hat{D}_{i j}\right) n_{j} \mathbf{m}_{j}$ where $\mathbf{m}_{j}=\left\langle\boldsymbol{\mu}_{i}\right\rangle$ are the local magnetizations. Specifically, in this approximation we have

$$
H_{\mathrm{eff}}=\sum_{i} n_{i}\left[-\mathbf{H}_{i} \boldsymbol{\mu}_{i}+E_{a}\left(\boldsymbol{\mu}_{i}\right)\right],
$$

and the free energy

$$
F=\left\langle H-H_{\text {eff }}\right\rangle-T \ln \operatorname{Tr} \exp \left(-\beta H_{\text {eff }}\right) .
$$

The microstructure is defined by the set of $n_{i}$. For the studies of simple configurations (e.g. for an ideal polytwinned stack with no defects, see below) this set may be prepared by hand. More realistic configurations may be obtained in microscopic simulations based on the master equation approach, ${ }^{9,10,13}$ where the configurational states of the alloy are described in terms of ensemble-averaged site occupations, or concentrations $c_{i}=\left\langle n_{i}\right\rangle$. Such configurations may be easily used in magnetic simulations, and in MFA we obtain

$$
F=-E_{J, D D}-T \sum_{i} c_{i} \ln \int d \hat{\mu}_{i} \exp \left[\beta\left(\mathbf{H}_{i} \boldsymbol{\mu}_{i}-E_{a}\left(\boldsymbol{\mu}_{i}\right)\right],\right.
$$

where $E_{J, D D}=\Sigma c_{i} c_{j} \mathbf{m}_{i}\left(-J_{i j}+\hat{D}_{i j}\right) \mathbf{m}_{j}$ is the total exchange and dipole-dipole energy, and $n_{i}$ are replaced by $c_{i}$ in $\mathbf{H}_{i}$. It is convenient to treat the contribution of the anisotropy energy perturbatively, since $E_{a}(\boldsymbol{\mu}) \ll T$.

Let us emphasize that the above form of $P$ is a reduced Gibbs distribution. The effective Hamiltonian contains effective parameters depending on the averages over dynamical variables, while the Gibbs distribution contains the Hamiltonian with actual interaction parameters. In particular, the generalized Gibbs distribution with the effective Hamiltonian and proper boundary conditions may describe inhomogeneous equilibrium states (e.g., the domain walls), while the standard Gibbs distribution may not.

In cases when MFA is inadequate (e.g., in frustrated antiferromagnets) one can use other statistical methods to calculate $F$, e.g., the cluster variation method ${ }^{14}$ or the cluster field method ${ }^{15}$ (although this will considerably complicate the calculations in the inhomogeneous case).

If magnetization $\mathbf{M}(\mathbf{r})$ slowly varies in space and is constant in magnitude, Eq. (5) reduces to the micromagnetic free energy $^{12}$ (see also Ref. 16). In this case all choices of $J_{i j}$ and $b_{i}$ are equivalent if they produce the same macroscopic properties $C$ (exchange constant) and $K$. In principle, the problem on the microstructural length scale may be addressed using micromagnetic methods with singularities of $C$ and $K$ at the twin boundaries (and defects, if present). However, for the studied magnets where $\delta$ is quite small and the characteristic microstructural length scales are of the order of 10 $\mathrm{nm}$, the continuous approximation has little computational advantage, which is offset by the need for cumbersome treat- ment of defects. It is easier and more natural to use the same microscopic approach for such simulations, where no special treatment is required for defects.

The method described above allows one to quickly find equilibrium configurations for simulation boxes with up to $\sim 10^{6}$ atoms. We used rectangular simulation boxes with periodic boundary conditions. The long-range dipole-dipole fields are computed using the Fourier transforms, and the demagnetizing effects associated with the surface of the sample are neglected.

We now explore the properties of MDWs in polytwinned magnets using the approach described above. It is convenient to use the body-centered tetragonal (bct) representation of the fcc lattice (with $c / a=\sqrt{2}$ and $c$ equal to the fcc lattice parameter). The equilibrium MDWs of two characteristic orientations, normal to (1 10$)$ and (001) planes, are shown in Fig. 1 for the CoPt model. Infinite stack of $c$ domains is assumed with ideal twin boundaries lying in the (110) planes. The $c$ domains have the same thickness $d=64 a_{\mathrm{bct}} \simeq 17 \mathrm{~nm}$ and are fully ordered (all $c_{i}$ are either 1 or 0 ). The easy axis $\mathbf{e}_{i}$ points along the local direction of the tetragonal axis, and $b_{i}=b$. For simplicity, only the transition-metal atoms are assumed to have the magnetic moment. Room temperature $T=0.4 T_{c}$ is assumed (for CoPt $T_{c}=720 \mathrm{~K}$ ), and the values of $b$ and $\mu$ are chosen so that MFA gives experimental roomtemperature values of $K=4.9 \times 10^{7} \mathrm{erg} / \mathrm{cm}^{3}$ and $\eta=0.082$ for CoPt. ${ }^{5}$ The parameters $J_{i j}$ for nearest and next-nearest neighbors were chosen as $J_{2} / J_{1}=2 / 3, J_{3} / J_{1}=1 / 6$; such choice makes the exchange constant $C$ isotropic.

The MDWs of both orientations shown in Fig. 1 have a peculiar feature: the DW segments located in adjacent $c$ domains are displaced in respect to each other. The reasons for this displacement may be understood from Fig. 2 where the structure of an intersection of a DW with a twin boundary is shown at atomic resolution. The displacement leads to the increase in $E_{\mathrm{DD}}$ due to the appearance of magnetically charged segments of $90^{\circ}$ DWs at the twin boundaries (central part of Fig. 2), but due to the small value of $\eta$ this increase is unimportant at the length scale of $\delta$. From the other hand, the displacement lowers the exchange energy at intersections because magnetization within each DW segment (shown by arrows at margins of Fig. 2) becomes parallel to that in the adjacent $c$ domain. The total exchange energy is lowest when the displacements alternate regularly in the stack (otherwise the magnetization would have to reverse within the DW segments); the dipole-dipole energy is also lower for alternating displacements because the magnetic charges at the twin-boundary $90^{\circ} \mathrm{DWs}$ in this case alternate in sign, and the MDW as a whole is magnetically uncharged and does not generate a long-range magnetic field. An important property of MDWs shown in Fig. 1 is that their segments are coupled only by magnetostatic forces which are relatively weak $(\eta \ll 1)$.

The orientation of the DW segments within the $c$-domains in the $(1 \overline{1} 0)$ oriented MDW is determined by two parameters: the anisotropy of the exchange constant $A_{C}$ $=C_{x} / C_{z}$ and the parameter $\xi=\eta d / \delta$. If $\xi \ll 1$, the DW orientation is completely determined by $A_{C}$. The surface tension of a DW is lowest when the DW aligns normal to the 
(a)
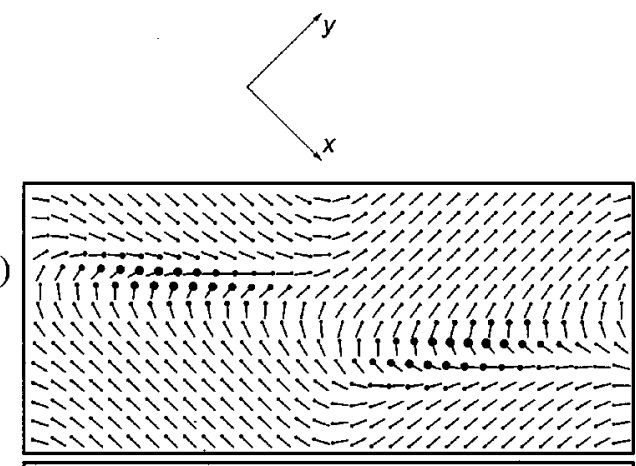

(b)

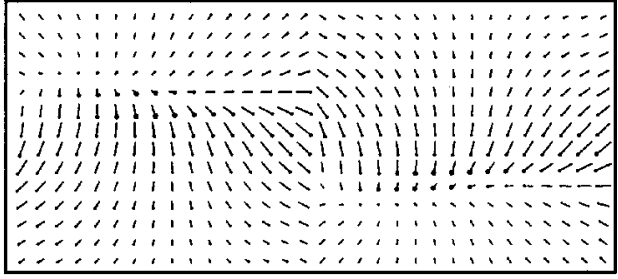

(c)

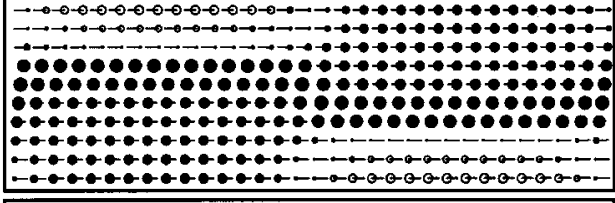

(d)

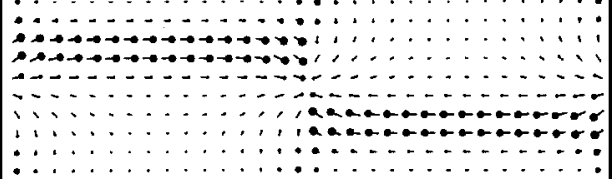

FIG. 1. Macrodomain walls in an ideal $X Y$ stack with $d=64 a_{\mathrm{bct}}$ oriented normal to: (a),(b) (1 10$)$; (c),(d) (001). Twin boundaries are vertical at edges and in the middle; axes at the top are shown for frames (a),(b). Panels (a),(c): magnetization $\mathbf{M}(\mathbf{r})$; (b),(d): dipole fields. Vectors are shown for unit cells on a square grid with a four-cell edge. The length of a stick (up to the center of the circle) is proportional to the vector projection onto the graph plane; the diameter of a full (open) circle, to the positive (negative) out-of-plane component. Small points at the end of long sticks show their direction. The simulation boxes had $512 \times 128 \times 1$ and $512 \times 128 \times 2$ bct cells for (11 10$)$ and (001) MDWs, respectively. Regions far from the MDW at top and bottom were removed to conserve space.

direction in which $C$ is lowest. The DW orientation is determined by competition of the surface tension and the total DW area. Similar considerations apply to an antiphase boundary in the polytwinned structure. ${ }^{17}$ If $\xi \gg 1$, the DW aligns parallel to the tetragonal axis minimizing the dipole energy, and $A_{C}$ is irrelevant. The former case is typical for $\mathrm{CoPt}$ and $\mathrm{FePt}$ at early stages of annealing, while FePd is typically in the intermediate $\xi \gtrsim 1$ region from the very onset of polytwinning. The (1 $1 \overline{1} 0)$ MDW shown in Figs. 1(a) and 1 (b) is in the crossover region with $\xi \sim 0.2$ and $A_{C}=1$.

In conclusion, we found the equilibrium structure of macrodomain walls in an ideal polytwinned magnet using a microscopic approach with the MFA expression for the inhomogeneous free energy (5). This structure turns out to be rather unusual, with separate domain wall segments coupled by magnetostatic forces. The resulting peculiarities of the hysteretic phenomena will be discussed elsewhere. ${ }^{18}$

The authors are grateful to H. Kronmüller, R. Skomski, and V. G. Vaks for useful discussions. This work was carried

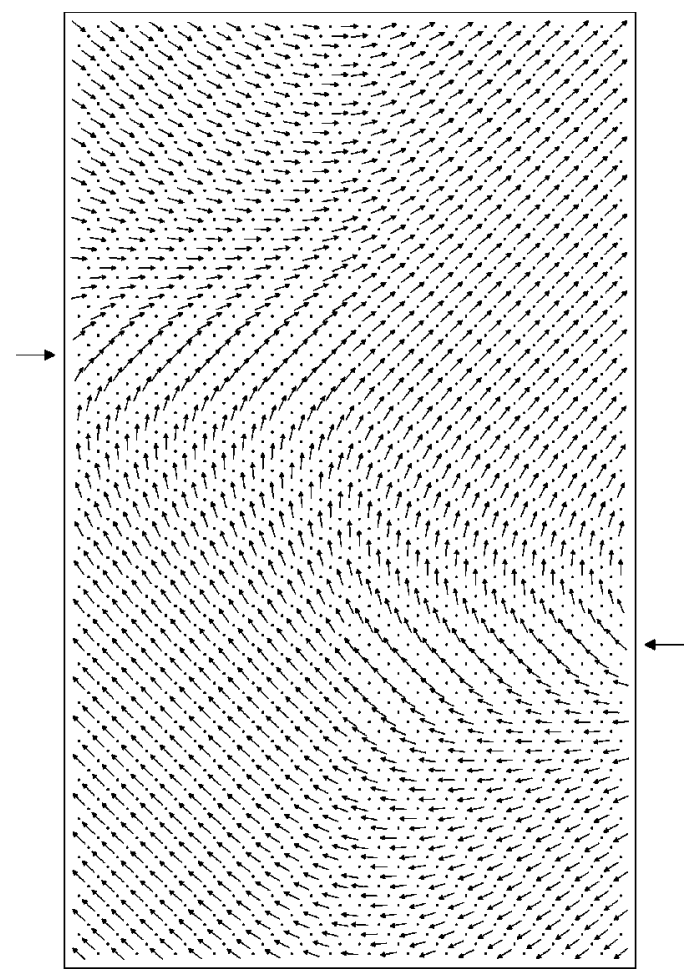

FIG. 2. Area of an intersection of MDW with a twin boundary. Arrows show $\mathbf{m}_{i}$ for sites occupied by magnetic atoms. Points show the positions of nonmagnetic atoms. Two adjacent atomic layers of the bct lattice are projected onto the plane of the graph. Arrows at margins show the approximate positions of DW segments at each side of the vertical twin boundary.

out at the Ames Laboratory, which is operated for the U.S. Department of Energy by Iowa State University under Contract No. W-7405-82. This work was supported by the Director for Energy Research, Office of Basic Energy Sciences of the U.S. Department of Energy.

${ }^{1}$ D. Goll and H. Kronmüller, Naturwissenschaften 87, 423 (2000).

${ }^{2}$ M. E. McHenry and D. E. Laughlin, Acta Mater. 48, 223 (2000).

${ }^{3}$ H. Kronmüller, R. Fischer, M. Seeger, and A. Zern, J. Phys. D: Appl. Phys. 29, 2274 (1996).

${ }^{4}$ J. Fidler and T. Schrefl, J. Phys. D: Appl. Phys. 33, R135 (2000).

${ }^{5}$ N. I. Vlasova, G. S. Kandaurova, and N. N. Shchegoleva, J. Magn. Magn. Mater. 222, 138 (2000).

${ }^{6}$ C. Leroux, A. Loiseau, D. Broddin, and G. Van Tendeloo, Philos. Mag. B 64, 58 (1991).

C. Yanar, J. M. K. Wiezorek, and W. A. Soffa, in Phase Transformations and Evolution in Materials, edited by P. Turchi and A. Gonis (The MMM Society, Warrendale, PA, 2000).

${ }^{8}$ L.-Q. Chen, Y. Wang, and A. G. Khachaturyan, Philos. Mag. Lett. 65, 15 (1992)

${ }^{9}$ K. D. Belashchenko, I. R. Pankratov, G. D. Samolyuk, and V. G. Vaks, J. Phys.: Condens. Matter 14, 565 (2002)

${ }^{10}$ V. G. Vaks, JETP Lett. 63, 471 (1996).

${ }^{11}$ L. D. Landau and E. M. Lifshitz, Statistical Physics, Part 1 (Pergamon, Oxford, 1980).

${ }^{12}$ A. Aharoni, Introduction to the Theory of Ferromagnetism (Clarendon, Oxford, 1996).

${ }^{13}$ K. D. Belashchenko and V. G. Vaks, J. Phys. Condens.: Matter 10, 1965 (1998).

${ }^{14}$ A. Finel, in Statics and Dynamics of Alloy Phase Transformations, NATO ASI Series B, Vol. 319, edited by A. Gonis and P. Turchi (Plenum, New York, 1994), p. 495

${ }^{15}$ V. G. Vaks and G. D. Samolyuk, Sov. Phys. JETP 88, 89 (1999).

${ }^{16}$ H. Kachkachi and D. A. Garanin, Physica A 291, 485 (2001).

${ }^{17}$ V. G. Vaks, JETP Lett. 73, 237 (2001).

${ }^{18}$ K. D. Belaschenko and V. P. Antropov, cond-mat/0110526 